\title{
CHAPITRE 16
}

\section{HORMONES ET IMMUNITÉ}

\section{INTRODUCTION}

Les corrélations entre systèmes endocrinien et immunitaire (constituant le cadre d'une endocrino-immunologie) ont été mises en évidence par de nombreux chercheurs.

\subsection{INTERRELATIONS THYMUS - GLANDES ENDOCRINES}

Schwartz, puis Deschaux ont mis en évidence les interrelations thymus-glandes endocrines en étudiant les effets de diverses ablations de glandes endocrines, l'action de leurs hormones sur "l'hormone thymique" (Schwartz, 1973 ; Comsa, 1979) ou ceux de la thymectomie ou des extraits thymiques sur ces glandes endocrines (Deschaux, 1980). Si la surrénalectomie provoque une hypertrophie thymique, l'hypophysectomie entraîne une involution du thymus (Schwartz, 1973). Les effets de la thymectomie ne sont évidents chez le rat que jusqu'à l'âge de 60 jours. Elle provoque un déséquilibre hormonal important, au niveau de l'axe hypophysosurrénalien (diminution de la teneur plasmatique en ACTH et de la corticostérone après une stimulation initiale) et hypophyso-gonadique (diminution de la concentration plasmatique de LH et de testostérone). La thymectomie néo-natale entraîne, en outre, une élévation du taux plasmatique de la GH (mais son efficacité est diminuée). La dysgénésie ovarienne, provoquée chez la souris ou le rat par une thymectomie néo-natale, est corrigée par des injections de thymosine.

\subsection{RôLE IMMUNITAIRE DU THYMUS}

Miller a montré, en 1961, que la thymectomie néo-natale s'accompagne d'une déficience immunologique à médiation cellulaire chez la souris ; puis des chercheurs ont mis en évidence les facteurs hormonaux d'origine thymique qui permettent la différenciation des lymphocytes pré-T en lymphocytes $\mathrm{T}$ et l'immunomodulation de leur activité :

- l' $\alpha$ 1-thymosine (ou plutôt la pro-thymosine $\alpha$ ) (A.L. Goldstein et coll., 1966) ;

- les thymopoiétines I et II (G. Goldstein, 1975);

- et la thymuline ou facteur thymique sérique (Bach et coll., 1972). 
Seuls, les deux derniers facteurs seraient spécifiques du thymus. Mais d'autres facteurs thymiques, qui n'ont pas encore été identifiés, interviennent certainement dans l'immunomodulation et l'acquisition de l'immunocompétence.

\subsection{AUTO-IMMUNITÉ ET PATHOLOGIE ENDOCRINIENNE}

Dans le domaine de la pathologie, l'étiologie auto-immune de nombreuses maladies est une réalité qui s'est imposée ces dernières années, en même temps que s'affirmaient les méthodes de détection et que s'approfondissaient les mécanismes pathogéniques de ces maladies. C'est en 1956 que Doniach et Roitt mettent en évidence des anticorps antithyroglobuline dans la thyroïdite de Hashimoto, que l'on considéra dès lors comme une maladie auto-immune ${ }^{1}$. La découverte du LATS (long acting thyroid stimulator) par Adams et Purves, la même année, (voir chap. 5.9) n'a pas suffi à l'époque à convaincre que la maladie de Basedow relevait du même mécanisme, d'autant que cet anticorps avait une action stimulante (et non pas neutralisante) sur la cellule thyroïdienne. On sait actuellement mieux comprendre ce paradoxe. Et de très nombreuses autres maladies endocriniennes s'avèrent relever d'un processus auto-immun : le diabète de type I (voir chap. 7, § 8.4.1), la maladie d'Addison (son autre étiologie, tuberculeuse, ayant presque disparu)...

\subsection{INTERRELATIONS HORMONES - CELLULES IMMUNITAIRES}

Les travaux plus récents mettent en évidence deux ordres de faits :

- les lymphocytes B ou T, les macrophages et éventuellement d'autres cellules intervenant dans la réponse immunitaire (mastocytes...) possèdent des récepteurs spécifiques à de nombreuses hormones. Celles-ci exercent sur les cellules immunitaires des effets modulateurs de leurs activités.

- les cellules immunitaires (lymphocytes, macrophages...) sécrètent des facteurs qui, pour certains, agissent sur des cellules endocrines, tandis que d'autres semblent voisins sinon identiques en structure à des hormones.

\section{RÉCEPTEURS SPÉCIFIQUES AUX HORMONES ET ACTION DES HORMONES SUR LES LYMPHOCYTES}

Les lymphocytes $\mathrm{T}$ et $\mathrm{B}$ possèdent des récepteurs aux hormones suivantes ${ }^{2}$ :

- prolactine (Russel et coll., 1984);

1 Ce qui n'était qu'un argument présomptif, et non une preuve, car les auto-anticorps peuvent être secondaires à l'altération cellulaire et non la cause de cette altération.

2 Mais, pour les œstrogènes, les récepteurs seraient présents sur les cellules épithéliales du thymus et non sur les thymocytes. 
- GH (Pandian et Talwar, 1971);

- prolactine (Russel et coll., 1985) ;

- opiacés : $\alpha$ - et $\beta$-endorphines (Gilman et coll., 1984);

- adrénaline et NA (Miles et coll., 1984);

- ACTH (Johnson et Torres, 1985) ;

et les lymphocytes $\mathrm{T}$ des récepteurs à :

- l'ADH (Johnson et Torres, 1985);

- l'acétylcholine (Shapiro, 1980);

- l'insuline (Murphy et coll., 1984);

- la substance P (Payan et Gœtzl, 1985);

- le VIP (O'Dorisio et coll., 1985) ;

- la met-enképhaline (Wybran et Schandené, 1986)...

\subsection{L'HORMONE DE CROISSANCE}

- Elle ne représente qu'un facteur de croissance adjuvant pour les lymphocytes.

- Elle est active en synergie avec T4 (Pierpaoli et coll., 1969). La thyroxine aurait un effet permissif sur la sécrétion et l'action de l'hormone thymique (Comsa et coll., 1974).

- L'addition de GH à des lymphocytes $\mathrm{T}$ de souris, in vitro, n'entraîne pas de prolifération significative (Snow et coll., 1981). Au contraire, si les lymphocytes ont été stimulés par la ConA (concanavaline A) ou la PHA (phytohémagglutinine), l'addition de GH inhibe la réponse blastogénique (Grossman et Rodelle, 1983).

- Toutefois, la réponse des lymphocytes aux lectines (incorporation de thymidine ${ }^{3} \mathrm{H}$ ) est GH-dépendante (Schimpff et Bozzola, 1984).

\section{2. $L^{\prime} A C T H$}

- Pour certains auteurs, elle joue un rôle modulateur de la croissance et de la différenciation des lymphocytes B (qui sont stimulés). Cette action in vitro nécessite la présence de BCGF (B cell growth factor) ou de l'IL2 (interleukine 2) (Alvarez-Mon, 1985) ;

- pour d'autres, c'est un inhibiteur puissant de la production in vitro d'IFN $\alpha$ (interféron $\alpha$ ) par des splénocytes de souris stimulées par des mitogènes (Johnson et coll., 1984), et elle supprime la réponse anticorps des splénocytes de souris aux GRM (globules rouges de mouton). 


\subsection{LES ENDORPHINES}

- L' $\alpha$-endorphine (comme les enképhalines) est un inhibiteur puissant de la réponse Ac aux GRM. $\beta$ - et $\gamma$-endorphines sont beaucoup moins actifs (Johnson et coll., 1982). L'effet inhibiteur de l' $\alpha$-endorphine est bloqué par la naloxone.

- La $\beta$-endorphine augmente la prolifération des lymphocytes T stimulée par ConA ou PHA, alors que l' $\alpha$-endorphine (comme les enképhalines) n'a aucun effet sur cette prolifération. Cet effet de la $\beta$-endorphine n'est pas supprimée par la naloxone. Aucun de ces opiacés endogènes n'a d'action sur la prolifération des lymphocytes B stimulés par un mélange dextran-LPS (Gilman et coll., 1982).

La $\beta$-endorphine augmente l'activité cytolytique et la production d'IFN (interféron) des cellules NK (natural killers).

\subsection{LA TSH}

- Elle augmente la réponse in vitro aux GRM (globules rouges de mouton) qui sont Ag thymodépendants ;

- elle augmente aussi la réponse in vitro à Brucella abortus-TNP (Ag thymoindépendant) (Kruger et Blalock, 1986).

\subsection{LA SOMATOSTATINE}

Elle a été testée in vitro sur des lymphocytes de rate, des plaques de Peyer et des ganglions mésentériques de souris stimulés par la ConA :

- elle diminue de 30 à $50 \%$ la synthèse d'ADN ;

- elle inhibe de 20 à $50 \%$ la sécrétion des IgA ;

- elle inhibe de 10 à $30 \%$ la sécrétion des IgM.

\section{6. $L^{\prime} \alpha-M S H$}

Elle inhibe l'effet de l'IL1 (interleukine 1) sur ses cellules cibles (elle serait un antagoniste endogène) concernant la prolifération des thymocytes de souris "in vitro" et la production des PgE par les fibroblastes, mais pas sur l'inhibition de la prolifération induite par l'IL2 des LT (lymphocytes T) cytotoxiques (Cannon et coll., 1986).

\subsection{LA PROLACTINE}

Elle intervient comme modulateur de la réponse des LT à la stimulation antigénique, réponse qui est inhibée par la bromocryptine. Le mode d'action de la cyclosporine A, qui est un immunosuppresseur puissant, peut s'expliquer par un effet compétitif avec la prolactine sur un même récepteur au niveau des lymphocytes T (Hiestand et coll., 1986). 
Ce tour d'horizon des effets des hormones hypothalamo-hypophysaires sur le système immunitaire permet de concevoir la réalité de l'impact du stress sur ce système, et aussi sa complexité. Si l'effet global sur les hormones paraît être une stimulation de la réponse immunitaire, il peut être compensé par l'effet inhibiteur des glucocorticoïdes (sur lesquels existe une littérature très abondante). Quant à l'adrénaline, certaines données seraient en faveur d'une inhibition des T suppresseurs (via des récepteurs $\alpha 2$ ) et d'une stimulation de l'activité cytotoxique (via des récepteurs $\beta$ ).

\section{FACTEURS SÉCRÉTÉS PAR LES CELLULES IMMUNITAIRES}

Les études ont été réalisées principalement par le groupe de Blalock au Texas. Le traitement des splénocytes de souris (probablement les macrophages) par des inducteurs de la sécrétion d'IFN $\alpha$ (interféron $\alpha$ ) entraîne la sécrétion d'ACTH-like et d'endorphin-like (Blalock et Smith, 1980-1981).

Ces facteurs sont semblables aux hormones hypophysaires du point de vue antigénique et activité biologique. Les cellules immunitaires seraient capables de sécréter non seulement des dérivés de la POMC, mais aussi de la TSH (Smith et coll., 1983), du VIP, de la somatostatine (Lygren et coll., 1984), et peut-être aussi de la gonadotrophine chorionique, de la GH, de la FSH et de la LH. Les cytokines des monocytes (par exemple l'interleukine 1 $\beta$ ) possèdent une puissante action stimulatrice sur l'axe hypothalamo-hypophyso-surrénalien (CRF 41-ACTH-corticoïde).

Si ces données sont confirmées, elles autorisent à concevoir un impact des cellules immunitaires sur le système des cellules endocrines. Ce rôle doit être toutefois limité, car il faut rappeler qu'une souris "nude" ${ }^{3}$ ne présente pas de désordres endocriniens considérables.

\section{STRESS ET IMMUNITÉ}

Les nombreux travaux qui mettent en évidence un lien entre le stress et le système immunitaire ont permis de développer une nouvelle discipline : la psycho-neuroimmunologie (Weinberg, 1994). On en trouvera des mises au point dans Baltrusch et coll. (1991), Cacciopo (1993), Stone et Boubjerb (1994).

3 Mutation qui entraîne une agénésie congénitale du thymus associée à l'absence de pelage. 


\subsection{BASES PHYSIOLOGIQUES ET MOLÉCULAIRES}

Le système nerveux central peut interagir avec le système immunitaire :

- via l'axe hypothalamo-hypophyso-surrénalien, mettant en jeu le CRF, l'ACTH et les glucocorticoïdes ;

- via l'innervation périphérique des organes lymphoïdes ;

- mais aussi par libération de neurotransmetteurs dans le sang.

Parmi ceux-ci, la substance $\mathrm{P}$, le vasoactive intestinal peptide (VIP) et les peptides opioïdes peuvent agir directement sur les lymphocytes (Payan et Goetzl, 1985 ; Morley et coll., 1987 ; Ruff et coll., 1988) ; en outre, des récepteurs pour de nombreux neurotransmetteurs ont été mis en évidence dans les lymphocytes humains circulants (Harper et coll., 1984 ; Bondy et coll., 1985 ; Rabey et coll., 1986 ; Eva et coll., 1989). C'est ainsi que le CRF est l'un des principaux agents des modifications immunitaires dans le stress puisqu'il peut stimuler l'ACTH et les endorphines non seulement au niveau de l'adénohypophyse, mais aussi à partir des lymphocytes (Smith et coll., 1986) et libérer ainsi la sécrétion de cortisol (Smith et coll., 1982).

- Une étude exhaustive sur l'altération de la fonction immunologique sous l'effet du stress a été réalisée par Rabin et coll. (1990). Ces auteurs ont recherché, sur deux lignées différentes de rats, l'effet de deux agents stressants, le choc électrique et le 2-déoxy-D-glucose (qui est un agent stressant métabolique). Les modifications portant sur les lymphocytes ont été observées dans le sang, la rate (pour les cellules NK) et les ganglions lymphatiques. Ils ont enfin déterminé le mécanisme de ces modifications, et en particulier les implications des stéroïdes surrénaliens et des opioïdes endogènes.

Les résultats observés ont été identiques quelle que soit la lignée utilisée. Les auteurs ont pu définir trois mécanismes différents : un mécanisme surrénaliendépendant à l'égard de la fonction mitogénique des lymphocytes sanguins périphériques, qui est déprimée (le retour à la normale nécessite 4 à 5 jours); un mécanisme surrénalien-indépendant dans le cas des lymphocytes spléniques, à récupération plus rapide ; une modification de la fonction des cellules NK (natural killers) de la rate par un mécanisme opioïde (antagonisé par la naltrexone).

- L'étude exhaustive rapportée par Cacciopo (1993) est le résultat d'une recherche commune à plusieurs équipes. La réponse de l'immunité cellulaire à un stress psychologique aigu se traduit par une baisse sensible de la fonction mitogénique des lymphocytes (fig. 16.1.a), par une élévation des $\mathrm{CD}^{+4}$ (et une baisse du rapport $\left.\mathrm{CD}^{+} / \mathrm{CD}^{+}\right)$, et une élévation importante du nombre et de la toxicité des cellules NK (fig. 16.1.b).

4 CD4 et CD8 sont des molécules glycoprotéiques; CD4 est liée aux molécules de classe II du complexe majeur d'histocompatibilité $(\mathrm{CMH})$, dans le cadre de la reconnaissance de la cellule présentatrice d'antigène; CD8 se lie aux molécules HLA (human leucocyte antigen) de classe I. 


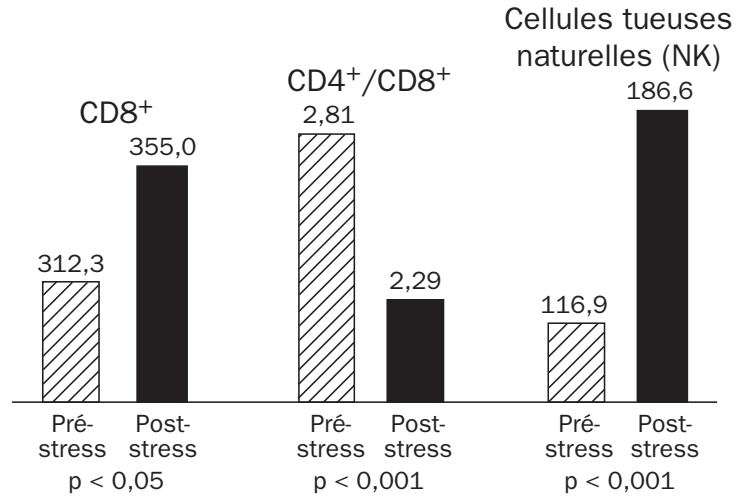

a - Réponse mitogénique à la ConA (concanavaline A) et à la PHA (phytohémagglutinine) - la réponse est exprimée en log des valeurs du comptage par minute

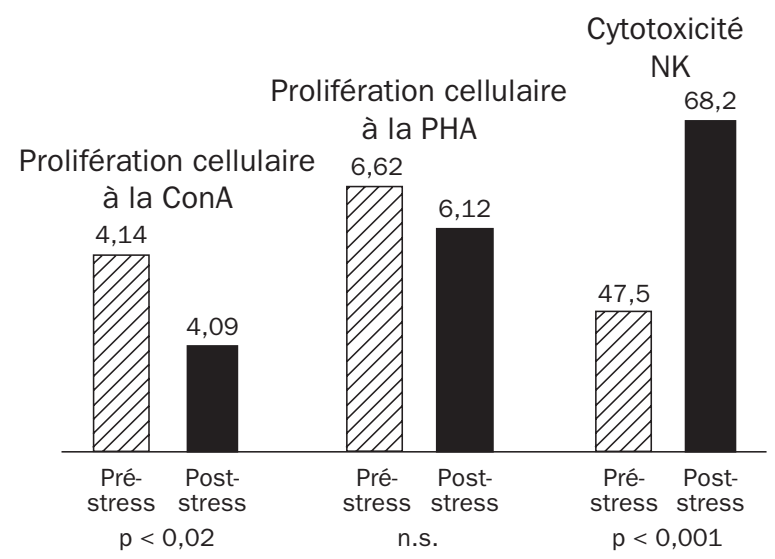

b - Réponse des différents types cellulaires - les chiffres représentent le nombre des cellules par $\mathrm{mm}^{3}$

Figure 16.1 - Réponse immunitaire cellulaire à un stress psychologique aigu

- L'étude biochimique des drogues utilisées pour traiter les symptômes pathologiques du stress peut constituer un outil d'étude des mécanismes impliqués dans les réactions du stress.

En particulier, les benzodiazépines (BDZ) semblent les plus utilisées pour traiter les manifestations d'anxiété, qui apparaissent liées au stress chronique chez l'homme. Ces benzodiazépines neutralisent en effet les modifications comportementales (Haefely et coll., 1981), neurochimiques (Rossetti et coll., 1990) et hormonales (Bizzi et coll., 1984) induites par le stress.

\subsubsection{Etude des récepteurs aux benzodiazépines (BDZ)}

Ferrarese et coll. (1993) se sont attachés à l'étude des récepteurs aux BDZ et à l'inhibiteur de liaison au diazépam (une benzodiazépine) relativement au stress. 
L'effet anxiolytique de BDZ est médié par un récepteur du système nerveux central (CBR ou central binding receptor) couplé à un récepteur GABA-A ${ }^{5}$; il agit par facilitation de la transmission gabaergique. Ces récepteurs sont nombreux dans le cerveau mammalien, particulièrement dans le cortex, le cervelet et les aires limbiques.

Les agonistes de ces récepteurs, tels que les BDZ, inhibent la libération de CRF en facilitant le tonus inhibiteur gabaergique, et agissent ainsi en immunosuppresseurs.

Mais les BDZ sont également actifs à un niveau périphérique sur une autre catégorie de récepteurs (PBR ou peripheral binding receptors) qui ont été trouvés dans tous les tissus de mammifères, parmi lesquels les cellules mononucléaires, la glande surrénale, les testicules et les ovaires. Ces récepteurs ne sont pas couplés à des récepteurs GABA-A, ils sont formés de deux sub-unités de respectivement 30 et $18 \mathrm{kDa}$.

Les auteurs ont étudié les récepteurs PBR dans les lymphocytes circulants de patients anxieux (suivant le DSM III-R, voir chap. 4 , § 10.5) et les effets de traitements aigus ou prolongés avec du diazépam. Le nombre des récepteurs, qui est diminué chez les patients anxieux, est restauré par le diazépam. Ces modifications paraissent spécifiques à l'anxiété.

Le rôle immunomodulateur joué par les récepteurs PBR semble doublé, in vitro, d'une action de contrôle sur la prolifération et la différenciation des cellules normales et cancéreuses. Le mécanisme biochimique qui lierait ces deux actions (immunomodulation et inhibition de la prolifération) est encore controversé, il passerait par une action sur la membrane externe de la mitochondrie et résulterait d'une inhibition à ce niveau de la stéroïdogenèse.

Les auteurs ont ensuite étudié les récepteurs CBR et PBR à la suite d'un stress (suite à un choc électrique sur la patte, ou une exposition au froid...). Les modifications observées ne sont pas univoques, elles dépendent de la nature de l'agent stressant ; les deux types de récepteurs peuvent même présenter des réponses de sens opposé.

\subsubsection{L’inhibiteur de liaison au diazépam et le stress}

La question qui s'est alors posée est : les ligands endogènes de ces récepteurs sont-ils impliqués dans la réponse au stress ? Parmi ceux-ci, l'attention s'est portée sur l'inhibiteur de liaison au diazépam (DBI), qui peut se lier aussi bien avec le CBR qu'avec le PBR.

On le trouve dans de nombreux organes (cerveau, foie, rein, surrénale...) de différentes espèces. Ce polypeptide est un précurseur de nombreux peptides qui se lient soit au PBR, soit au CBR, et qui sont des neuropeptides biologiquement actifs.

Il a été étudié chez des rats stressés et des patients anxieux.

5 GABA est un neurotransmetteur exerçant une action inhibitrice via l'activation de son récepteur (A) qui est un canal $\mathrm{Cl}^{-}$, et une relation stimulatrice via son récepteur (B). 
- Chez le rat stressé (stress aigu provoqué par le bruit) : élévation du DBI au niveau de l'hippocampe et de la glande surrénale, de façon très précoce, parallèlement au niveau de la corticostérone plasmatique. On peut supposer que le DBI est le médiateur de l'action stéroïdogène de l'ACTH au niveau de la glande surrénale.

- Chez les patients anxieux : diminution (-40\%) du taux de DBI dans les lymphocytes (due à un "turn-over" augmenté en faveur de petits peptides plus actifs ?) ; ce taux s'élève après un traitement prolongé par le diazépam.

En définitive, de nombreux arguments permettent de supposer que le DBI est un médiateur des effets centraux et périphériques du stress, en se liant aux deux types de récepteurs aux benzodiazépines.

\subsection{ETUDES EXPÉRIMENTALES ET CLINIQUES}

Divers travaux permettent de conforter l'hypothèse que certains événements de la vie exercent une influence sur l'évolution d'un cancer. C'est le cas des travaux qui, dans le cadre de l'immunomodulation, démontrent l'influence du psychisme en général, et du stress en particulier, sur notre système immunitaire (Villemain, 1989 ; Ferrarese et coll., 1993).

\subsubsection{L'expérimentation animale}

Chez les animaux de laboratoire, il apparaît que, si le stress aigu et le stress chronique peuvent se manifester de façon opposée quant à plusieurs paramètres du stress, leur effet sur le système immunitaire est habituellement le même, c'est-à-dire une inhibition de ce système (Borg et Moller, 1978 ; Armario et coll., 1984 ; Goodkin et coll., 1986 ; Lahiri et Banerjee, 1986 ; Steplewski et Vogel, 1986 ; Dorian et Garfinkel, 1987).

Mais c'est une vision trop simpliste des choses, il faut préciser, lorsqu'on rapporte ces expériences, quelles modalités de la réponse immunitaire sont impliquées.

\section{Le stress aigu}

Le stress aigu diminue l'immunité (Rasmussen, 1969 ; Solomon, 1969 ; Keller et coll., 1981 et 1983).

Chez des souris soumises à des chocs électriques ou à des sons intenses, on observe un rejet diminué des greffes hétérologues (tissus d'animaux différents), une plus grande sensibilité aux virus pathogènes et, en cas d'infection, une diminution de la synthèse d'interféron. Les chocs électriques provoquent également une diminution de l'activité lymphocytaire en réponse aux mitogènes (Laudenslager, 1983), ainsi que de la production d'anticorps spécifiques (Laudenslager, 1988).

Le retentissement des agressions contre l'organisme dépend de la capacité de contrôle de l'agression. Il devrait en être de même pour les effets du stress sur l'immunité et c'est effectivement ce que l'on observe. 
En 1988, Mormède et coll. ont montré sur des rats soumis à des chocs électriques que la possibilité de contrôle de la situation (ici un évitement) entraîne, par rapport à des rats qui n'ont pas cette possibilité, une prolifération lymphocytaire plus élevée mais une production d'anticorps plus basse (fig. 16.2).

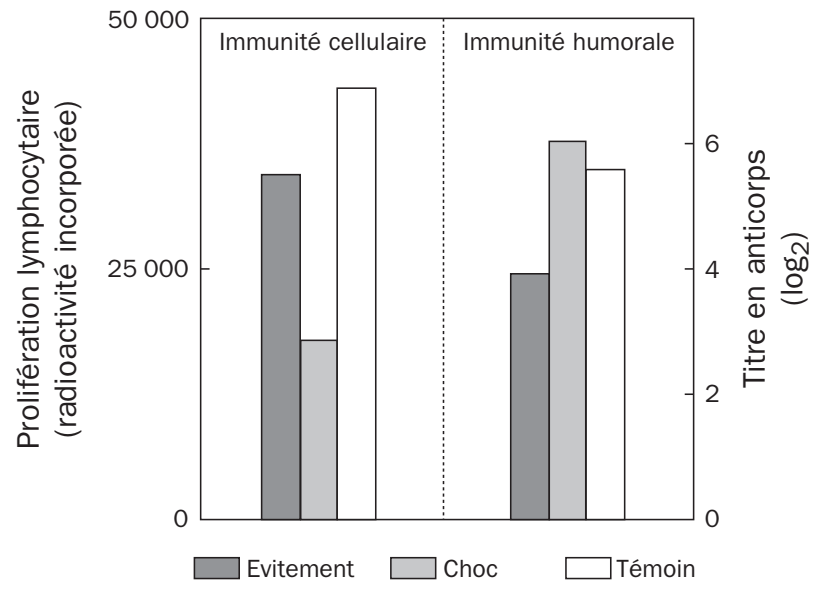

Figure 16.2 - Influence de la possibilité de contrôler le choc électrique

sur l'immunité cellulaire et humorale du rat (d'après Mormède et coll., 1988)

Des rats ayant appris à éviter des chocs électriques (évitement) sont comparés à des rats soumis aux mêmes chocs, mais sans possibilité d'évitement (choc), et à des animaux témoins sans choc (témoin), après dix séances de conditionnement. L'immunité cellulaire est mesurée par la prolifération lymphocytaire en réponse à un mitogène, tandis que l'immunité humorale est estimée par le titre en anticorps contre des globules rouges de mouton injectés au $5^{e}$ jour de l'expérience.

La plupart des expériences utilisent ainsi des agents stressants physiques qui ont l'avantage de la commodité. Les résultats sont parfois contradictoires. Mais leur caractère artificiel doit faire préférer des agressions survenant dans un contexte social. Ainsi, chez les rongeurs, diverses expériences démontrent que les animaux dominés présentent une diminution des réponses d'immunité cellulaire et humorale (Raab et coll., 1986).

En revanche, chez le Singe, la rupture de l'attachement entre le jeune et ses compagnons de captivité, si elle provoque une immunodépression de certains aspects de la réponse immunitaire (nombre des lymphocytes et des éosinophiles circulants, taux sériques d'immunoglobulines $\mathrm{G}$ ), favorise au contraire d'autres fonctions immunitaires : activité hémolytique du complément, action oxydative des macrophages (Reite et coll., 1981 ; Coe et coll., 1987).

\section{Le stress chronique}

Si le stress aigu est dépresseur de l'immunité cellulaire, les situations de stress chronique ou de stress répété peuvent au contraire, dans certaines conditions, entraîner une stimulation de l'immunité : augmentation de la prolifération lymphocytaire, de l'activité NK et de la réponse anticorps (Dunn, 1989). C'est dire qu'il faut être 
prudent dans les interprétations des relations entre stress et pathologie, d'autant que les résultats expérimentaux dépendent du type de facteur de stress utilisé, de même que de l'espèce animale étudiée.

Kort (1994) fait une étude détaillée des effets du stress chronique sur le système immunitaire et la carcinogenèse expérimentale chez le rat. Comme le fait remarquer cet auteur, il est difficile de réaliser un stress chronique. En effet, un agent stressant prolongé entraîne habituellement une adaptation et, s'il est peu intense, une habituation (voir Borg et Moller [1978] dans le cas d'un bruit). Aussi Kort et son groupe (Kort et Weijma, 1982 ; Kort et coll., 1986a et b) ont-ils préféré utiliser un stress physiologique inévitable, un stress par modification du rythme lumièreobscurité. Dans ces conditions, ils observent toujours une inhibition du système immunitaire.

\subsubsection{Stress et immunité chez l'homme}

On trouve généralement une diminution des défenses immunitaires chez des individus stressés.

On peut en fournir plusieurs exemples : la dépression, le deuil, le stress induit par un examen, la perte du travail. Un certain nombre de ces observations sont rapportées par Mazet (1986) ou Galinowski (1993).

\section{La dépression}

Les observations faites au cours de la dépression mettent en évidence des modifications significatives de la défense immunitaire. On en trouvera une étude détaillée dans la thèse de Maffre (1995). Dès 1973, de nombreux auteurs (Shopsin et coll., 1973 ; Johnston et Whaley, 1975 ; Deberdt et coll., 1976 ; Cappel et coll., 1978) rapportaient que la fonction lymphocytaire était altérée durant la phase aiguë de la maladie dépressive.

En comparant des sujets hospitalisés pour un épisode dépressif majeur, avec une population contrôle, le groupe de Stein (Schleifer et coll., 1984 et 1985) met en évidence une immunodépression qui porte à la fois sur l'immunité cellulaire et l'immunité humorale, tandis que les taux de cortisolémie sont augmentés. Des expériences ultérieures ont permis de conclure que l'hospitalisation par elle-même ne peut être à l'origine de cette inhibition immunitaire.

A la même époque, Kronfol et coll. (1983) font état d'une corrélation significative entre l'intensité de la dépression, mesurée par l'échelle de Hamilton (1967), et l'inhibition de la réponse lymphocytaire à un agent mutagène, le pokeweed ou PWM (fig. 16.3). Un an plus tard, en 1984, Kronfol et coll., partant de la constatation que la dépression primitive est souvent associée à une hyperactivité de l'axe hypothalamohypophyso-surrénalien, et donc à une hypercortisolémie (Sachar, 1975 ; Schlesser et coll., 1980), observent une diminution significative du nombre des lymphocytes chez 177 patients déprimés non-traités. 


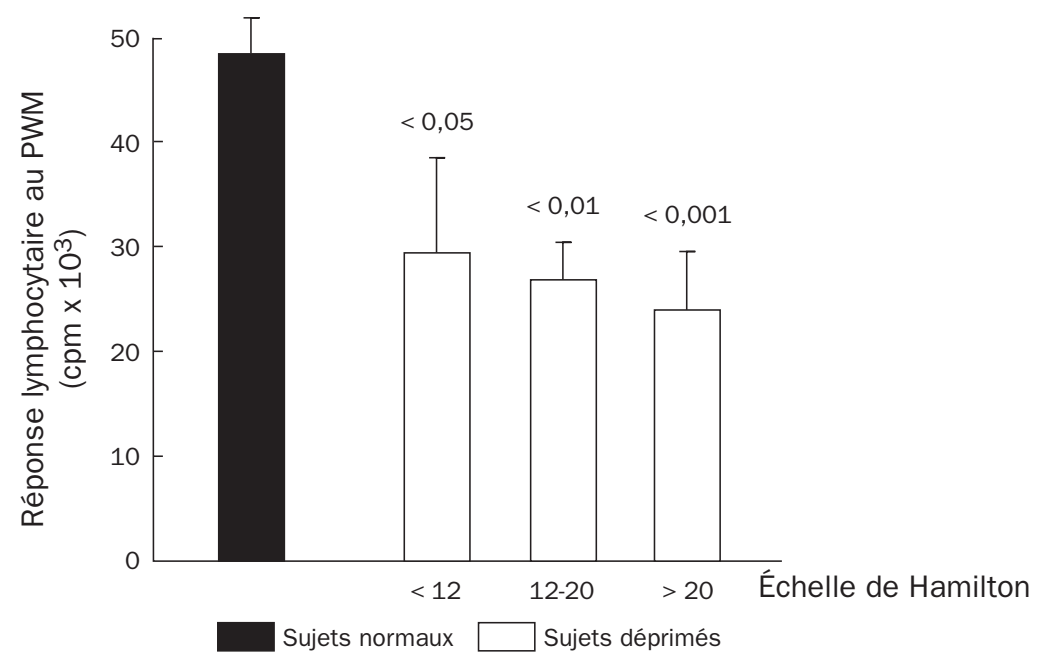

Figure 16.3 - Corrélation entre l'intensité de la dépression et l'inhibition de la réponse lymphocytaire (d'après Kronfol, 1983)

Shekelle et coll. (1981), dans une étude prospective portant sur 2020 hommes suivis pendant 17 puis 20 ans (Persky et coll., 1987), mettent en évidence une augmentation significative $(\times 2)$ de l'incidence des maladies néoplasiques chez des sujets présentant un score élevé de dépression, révélé au début de l'étude par le test MMPI ${ }^{6}$.

Cette conclusion n'était pas partagée par Kronfol qui exprimait, en 1983, que la relation entre les anomalies biologiques observées chez les déprimés et la plus grande vulnérabilité des déprimés aux infections et au cancer restait à établir. Et, effectivement, Zonderman et coll. (1989), dans une étude extensive sur 3980 sujets de plus de 55 ans, commencée en 1986 et comportant une mesure psychométrique des symptômes dépressifs à l'aide des échelles CES-D (Center for epidemiologic studies depression) et GWB-D (general well-being Schedule), dont 3814 (96\%) ont été revus à un deuxième check-up, concluent à l'absence de corrélation significative entre les symptômes dépressifs et le risque de morbidité ou de mortalité cancéreuse.

\section{Le deuil}

Des recherches ont été faites sur les effets de la perte d'un conjoint ou d'un enfant sur le système immunitaire. On doit à Bartrop et coll. (1977) la première étude objectivant un déficit immunitaire fonctionnel chez des sujets ayant perdu leur conjoint. On peut observer dans un délai de 2 à 6 semaines une diminution significative de la réponse proliférative lymphocytaire à deux agents mutagènes (stimulation par la PHA ou phytohémagglutinine et la ConA ou concanavaline A), c'est-à-dire de l'immunité cellulaire retardée. En revanche, il n'a pas été retrouvé de différence en ce qui concerne le nombre de lymphocytes $\mathrm{B}$ ou $\mathrm{T}$, la concentration d'immunoglobines, la

6 “Minnesota Multiphasic Personality Inventory" test. C'est un questionnaire destiné à quantifier des données attachées à une personnalité et à en faire une analyse statistique. 
présence d'anticorps, ni le taux des différentes hormones : cortisol, prolactine, hormone de croissance ou hormones thyroïdiennes.

Schleifer et coll. (1983) étudient quinze sujets dont la femme souffre d'un cancer à la période terminale. La comparaison entre leur système immunitaire avant le décès de leur épouse et dans les 2 mois qui ont suivi montre une diminution significative de trois tests immunitaires fonctionnels (les trois agents mutagènes sont la PHA, la ConA et le pokeweed). Mais ils ne relèvent aucune différence quant au nombre relatif ou absolu des lymphocytes B ou T.

Comme le fait remarquer Bonfils (1993), "si le décès d'un proche est assimilé, dans une certaine mesure, à une perte, il serait alors logique qu'un rôle déclenchant identique puisse être objectivé dans certaines situations vécues comme des pertes symboliques, telles que divorces, ruptures, licenciements, pertes monétaires". Toute proportion gardée, c'est bien ce que l'on a pu observer dans le stress conjugual (divorce), familial (décès du conjoint) ou professionnel où l'on observe une diminution des réponses d'immunité cellulaire et une augmentation de l'immunité humorale à l'égard de virus déjà présents dans l'organisme, comme le virus de l'herpès (Glaser et coll., 1987 ; Kiecolt-Glaser et coll., 1988).

Spratt et Denney (1991) trouvent, chez des parents d'enfant brutalement décédé, une perturbation durable des lymphocytes T-suppresseurs (qui sont diminués), des Thelpers (qui sont augmentés), mais pas de modification du taux de la cortisolémie.

Toutefois, Martin-Du Pan fait remarquer (1991) que les modifications immunitaires observées après un deuil sont beaucoup plus faibles que dans le SIDA, ou même chez les séropositifs.

\section{Les examens}

Quarante étudiants en médecine ont été testés 6 semaines avant les examens de fin d'année et juste le 1er jour des examens (voir fig. 16.4) : on a pu observer une forte réduction de la production d'interféron leucocytaire et une diminution du nombre et de l'activité des cellules NK (Glaser et coll., 1986).

\section{Tableau 16.1 - Influence des examens sur la production d'interféron leucocytaire et l'activité des cellules NK}

(d'après Glaser et coll., 1986)

\begin{tabular}{|l|c|c|c|}
\hline \multirow{2}{*}{} & $\begin{array}{c}\text { Interféron leucocytaire } \\
\text { (unités / } \mathrm{ml} \text { ) }\end{array}$ & \multicolumn{2}{|c|}{ Cellules tueuses naturelles (NK) } \\
\cline { 3 - 4 } & 2000 & $\begin{array}{c}\text { Pourcentage } \\
\text { circulant }\end{array}$ & $\begin{array}{c}\text { Activité } \\
\text { cytolytique }\end{array}$ \\
\hline Ligne de base & 80 & 16,3 & $45 \%$ \\
Examens & 9,1 & $30 \%$ \\
\hline
\end{tabular}

Les quarante étudiants en médecine ont été testés 6 semaines avant les examens de fin d'année (ligne de base) et le $1^{\text {er }}$ jour des examens. 
Dans une autre étude, Jemmott et coll. (1983) ont mesuré la sécrétion d'immunoglobulines dans la sécrétion salivaire de 64 étudiants en chirurgie dentaire, et trouvé que la sécrétion en IgA varie notablement :

- selon que l'examen représente une étape capitale du curriculum (haut stress) ou au contraire un contrôle de routine (bas stress) ;

- et selon la personnalité des étudiants, la sécrétion étant plus élevée chez ceux qui recherchent une relation interpersonnelle chaleureuse, et plus basse chez ceux qui réfrènent leur volonté de pouvoir (fig. 16.4).

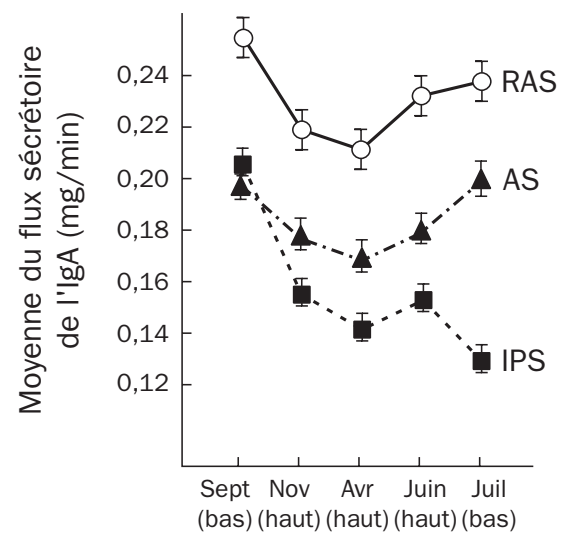

Figure 16.4 - Flux sécrétoire des IgA salivaires d'étudiants, selon le niveau haut ou bas du stress de l'examen et la personnalité de l'étudiant

(d'après Jemmot et coll., 1986)

As correspond à l'ensemble des étudiants, RAS à ceux qui recherchent une relation interpersonnelle chaleureuse et IPS à ceux qui réfrènent leur motivation de pouvoir.

\section{Autres causes de stress}

- Les troubles paniques (d'après la définition du DSM III), qui peuvent être d'ailleurs déclenchés par un facteur de stress, s'accompagnent d'une baisse significtive des IgA sériques (Ramesh et coll., 1991). Mais la validité de ce test ayant été critiquée (Stone et coll., 1977), la critique s'applique donc également à l'expérience précédente.

- L'effet de la perte de travail est un exemple de stress chronique. Il a été étudié chez des femmes qui avaient perdu leur emploi 9 mois avant le début de cette étude (Brenner, 1979). L'effet n'en était que psychologique car ces femmes avaient reçu une allocation correspondant à $90 \%$ de leur salaire. Le résultat observé était une diminution de la réponse à la PHA (phytohémagglutinine) et au PPD (dérivé protéique purifié de la tuberculine).

- Un autre exemple de stress chronique concerne les habitants de Three Mile Island, lieu d'un accident d'une centrale atomique en 1979. Ces habitants vivaient dans la peur des séquelles concernant leur santé et la crainte de la réouverture de la centrale. Ils ont présenté une réduction des lymphocytes $\mathrm{B}$, des cellules T suppressives et cytotoxiques, et des cellules NK (McKinnon et coll., 1989). 


\section{AUTO-ANTICORPS ET ENDOCRINOPATHIES AUTO-IMMUNES}

Dans le cadre de ces endocrinopathies, la relation entre système immunitaire et cellules endocriniennes n'a rien de spécifique, et il faut rechercher dans une prédisposition du "terrain", c'est-à-dire au niveau de gènes du système HLA ou dans la spécificité de l'agent causal ${ }^{7}$ (virus), la raison de l'atteinte du système endocrinien, lorsque celle-ci est isolée. Dans d'autres cas, l'endocrinopathie ne représente qu'un symptôme auto-immun parmi d'autres, elle n'est alors qu'une des expressions d'un dérèglement de la coopération/régulation des cellules immunitaires.

\subsection{LES AUTO-ANTICORPS - LE RÉSEAU IDIOTYPIQUE}

Il s'agit d'anticorps dirigés contre des constituants cellulaires d'un individu. Ces constituants antigéniques sont en général des composants biochimiques de la cellule : thyroglobuline, myéline ou des éléments membranaires, cytoplasmiques, microsomiaux ou nucléaires qu'on ne retrouve pas normalement dans la circulation. Ce peuvent être aussi des cellules entières, telles les spermatozoïdes, cellules qui n'existaient pas à la naissance et qui sont normalement élaborées puis éliminées à l'intérieur d'une cavité épithéliale fermée, et normalement sans contact avec le système immunitaire.

On pensait que l'apparition de ces auto-anticorps constituait un dérèglement pathologique de la régulation immunitaire. On sait qu'en fait l'organisme peut sécréter normalement, mais en très faible quantité, ces auto-anticorps.

Un exemple en est donné par le réseau idiotypique (fig. 16.5). Au départ hypothèse élaborée par Oudin (1968) puis Jerne (1984), elle a, depuis, reçu de nombreuses confirmations qui ont valu à Jerne le prix Nobel 1984. Soit un anticorps (Ab1) avec son idiotype, constitué par les parties variables des chaînes lourdes et légères (Fab) et qui porte des déterminants appelés idiotopes. Ces idiotopes peuvent être à l'intérieur ou à l'extérieur du site de liaison. Ceux qui sont à l'intérieur constituent le paratope, qui est stériquement complémentaire du site antigénique ou épitope. Selon Jerne, l'anticorps est susceptible d'induire des anticorps anti-idiotypes (Ab2), qui sont euxmêmes contrôlés par des anti-anti-idiotypes (Ab3).

L'anticorps anti-idiotype est en quelque sorte un auto-anticorps et c'est la raison pour laquelle son existence, compte tenu aussi de la sensibilité insuffisante des méthodes de dosage des anticorps à l'époque où Jerne a exprimé son hypothèse, était controversée. Dans la mesure où l'anti-idiotype est dirigé contre des idiotypes situés à l'intérieur du paratope, il est clair qu'il peut jouer le rôle de l'antigène d'origine, dont

7 Elle repose sur la similitude entre les molécules membranaires de la cellule endocrine et celles de l'enveloppe du virus. 
il sera une "image interne". Si cet antigène d'origine est une hormone, l'anti-idiotype est capable d'interagir avec le récepteur à l'hormone, pour lequel il se comportera comme un anticorps antirécepteur :

- soit bloquant (par exemple le diabète insulinorésistant). Il fonctionne comme antagoniste du site récepteur ;

- soit stimulant (par exemple la maladie de Basedow). Il se comporte alors comme un agoniste sur le site récepteur.
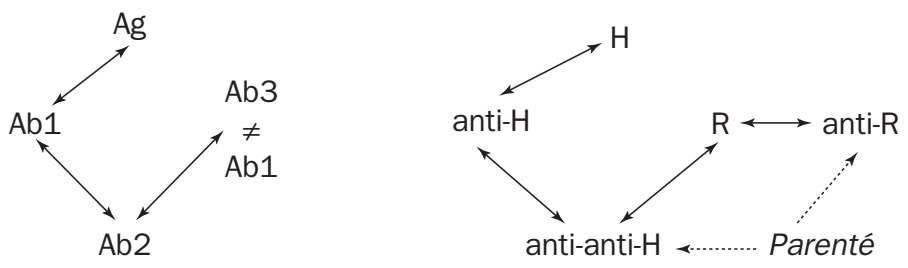

Figure 16.5 - Le réseau idiotypique de Jerne

\subsection{LA MALADIE AUTO-IMMUNE}

La maladie auto-immune correspond soit à des auto-anticorps sécrétés en quantité anormale (maladie auto-immune humorale), soit à une présence active de lymphocytes cytotoxiques dirigés contre les cellules de l'organisme (maladie auto-immune cellulaire). Ces deux types de dysfonctionnement auto-immun peuvent, bien sûr, être associés.

Les endocrinopathies auto-immunes, isolées ou associées, comprennent l'hypoparathyroïdie, l'hypothyroïdie primitive, la maladie de Graves-Basedow, la thyroïdite de Hashimoto, l'insuffisance surrénale chronique, l'hypogonadisme primitif, le diabète de type I, et même certains déficits hypophysaires isolés.

Maladie d'Addison auto-immune et maladie de Graves-Basedow représentent deux exemples différents d'endocrinopathie auto-immune.

- Dans les pays développés, la maladie d'Addison est liée deux fois plus souvent à une pathologie immune qu'à la tuberculose. On retrouve souvent (mais pas toujours) des anticorps anticortex surrénal. Une autre endocrinopathie lui est associée dans environ $1 / 3$ des cas, la surrénale ne représentant pas alors nécessairement la première atteinte endocrinienne. La maladie auto-immune entraîne dans ce premier exemple une insuffisance sécrétoire, c'est la conséquence la plus habituelle de l'atteinte auto-immune. On retrouve ce phénomène dans la thyroïdite autoimmune d'Hashimoto ${ }^{8}$, qui se traduit par un goître hypofonctionnel et pour

8 Le goître d'Hashimoto est l'une des trois formes distinctes de ce que l'on appelle la thyroïdite auto-immune, les deux autres formes étant le myxœdème spontané de l'adulte et la thyroïdite atrophique asymptomatique. 
laquelle on retrouve presque toujours une cytotoxicité à $\mathrm{T}$ cytotoxiques et parfois une ADCC (antibody dependant cytotoxicity).

Il est probable que la rupture de l'autotolérance résulte d'une prédisposition génétique HLA-dépendante (souvent DR3 et DR4, parfois B8). Elle est accompagnée dans le cas de la thyroïdite auto-immune, qui a été étudiée, d'un dérèglement du rapport entre les cellules $\mathrm{T}$ helper et $\mathrm{T}$ suppressives, et d'un défaut de fonctionnement de la cellule thyroïdienne.

- La maladie de Graves-Basedow est bien due à des anticorps, mais des anticorps immunostimulants. Les immunoglobulines sont des antirécepteurs à la TSH et agiraient soit comme agonistes de ces récepteurs, soit par une action sur la configuration du récepteur, ou d'une structure membranaire qui lui est associée. 
